\title{
Effect of Grafting Technique on Productivity and Quality of Cantaloupe under Saline Irrigation Water
}

\author{
Zaki ME' , Nadia S Shashak ${ }^{1}$, FA Abo Sedera ${ }^{1}$, AA Glala ${ }^{2}$, SA Saleh ${ }^{2 *}$ and AS Mohamed ${ }^{2}$ \\ ${ }^{1}$ Horticulture Department, Benha University, Egypt
}

${ }^{2}$ Horticultural Crops Technology Department, National Research Centre, Egypt

Received: 眥April 03, 2018; Published: 䤈April 13, 2018

*Corresponding author: SA Saleh, Horticultural Crops Technology Department, National Research Centre, Egypt

\begin{abstract}
The high salinity of the irrigation water is the biggest challenge facing horizontal expansion of vegetable cultivation especially in the new reclaimed land. The high salinity of the irrigation water is of a deleterious effect on the cantaloupe production. Thus, this experiment was carried out under greenhouse conditions during 2015 and 2016 autumn seasons in Moshtohor, Kalyobiya Governorate, Egypt to investigate the possibility of using grafting technique to ameliorate the negative effects of high salinity of irrigation water on cantaloupe productivity and its quality. Two commercial cultivars "Ideal and Veleta" were used as scion while Cobalt and Strong-Tosa were used as rootstocks. A modified tongue approach grafting method was used, and then seedlings were exposed to four salinity levels [0.8 (non-saline control), 3.9, 7.1 and $10 \mathrm{dSm}^{-1}$ ]. The results showed that all investigated factors "salinity levels, cultivars and rootstocks" significantly affected cantaloupe productivity and quality. Where, the medium salinity level $(3.9 \mathrm{dS} / \mathrm{m})$ resulted in the highest early yield, fruits number and total yield compared to all other salinity levels while the total yield decreased by $39.7 \%$ with increasing salinity levels up to $10 \mathrm{dS} / \mathrm{m}$. Whereas, graft combination of Ideal/Strong-Tosa increased the total yield by 53.1, 85.5, 43.8 and $1.4 \%$ at different salinity levels: control, $3.9,7$ and $10 \mathrm{dS} / \mathrm{m}$, respectively compared to the nongrafted plants of Ideal at non-saline control. This percentage was 38.4, 41.5, 19.3 and decline 13.1\% with Veleta/Cobalt compared to the non-grafted plants of Veleta at non-saline control. Generally, when cantaloupe plants have to be irrigated with high salinity of irrigation water, it is recommended to cultivate grafted seedling resulted of Veleta/Cobalt and Ideal/Strong-Tosa where these plants resulted the highest benefit and income compared with those on its own roots (non-grafted plants) under saline conditions.
\end{abstract}

Keywords: Cucumis melo; Cantaloupe; Grafting; Rootstock; Scion; Irrigation water and salinity

\section{Introduction}

Cantaloupe (Cucumis melo L.) is a high economic vegetable crop in many countries including Egypt. It is grown in practically every country in the world under outdoor fields or greenhouses. The cultivated area of cantaloupe in Egypt is 66,434 feddan $(4200 \mathrm{~m} 2)$ with total production of 846,936 tons and an average of 12.749 ton/fed. Ministry of Agric, Egypt, 2015. The most important problems facing horizontal expansion of cantaloupe in greenhouses or in open fields are the high salinity of the irrigation water or soil especially in the new reclaimed lands. As well as the recurrence of agriculture in greenhouses increases the soil salinity and thus reduces the vertical production of cantaloupe. In addition, cantaloupe is moderately salt tolerant, it has been determined that salinity causes several kinds of damage such as growth inhibition [1-3], yield and quality losses [4-6]. This leads us to use some newly trends to mitigate these negative impacts. The grafting technique is one of the most modern trends used to improve the productivity of vegetable plants, especially under adverse environmental conditions. Grafted vegetables onto resistant rootstocks offers numerous advantages on growth and yield, i.e., tolerance to salinity stress [7-10], increase yield and fruit quality in many crops such as melon [11] and watermelon [12,13]. Accordingly, the present study was conducted to investigate the possibility of using the grafting as a new promising technique for ameliorate the negative effects of the high salinity of the irrigation water on cantaloupe productivity and its quality. 


\section{Materials and Methods}

This investigation was carried out in a private farm in Moshtohor village, Kalyobiya Governorate, Egypt during 2015 and 2016 autumn seasons to study the response of yield productivity and fruits quality of grafted and non-grafted cantaloupe plants to different irrigation water salinity levels. The soil was clay with $\mathrm{pH}$ of 8.0 and EC of $1.3 \mathrm{dS} / \mathrm{m}$. Two commercial cultivars Veleta $R Z$ and Ideal (MG739) were grafted on the Cobalt RZ and Strong-Tosa rootstocks using modified tongue approach grafting method. The grafted and non-grafted seedlings were transplanted under net house condition, on the 21st of July in both investigation seasons. The plants were transplanted on one side of ridges $1.5 \mathrm{~m}$ width, at $50 \mathrm{~cm}$ apart. Four irrigation water salinity levels were applied [0.8, 3.9, 7.1 and $10 \mathrm{dSm}^{-1}$ ] by adding $\mathrm{NaCl}$ to the used underground water. A split split-plot designed was adopted, with three replicates where, salinity levels were placed in main plots, meanwhile cultivars "scions" in subplots and rootstocks in sub-subplot.

The yield of the first tow pickings was considered as early yield as well as number of fruits per plant and total yield per plant (g.) were calculated in the end of the growing season. The fruit length and diameter were measured to calculate fruit shape index (fruit length/fruit diameter) and. Finally, average fruit weight (g), flesh thickness of fruit $(\mathrm{cm})$ and seed cavity diameter $(\mathrm{cm})$ as well as total soluble solids percentage (AOAC, 1990) were measured. Data were subjected to the statistical analysis by the method of Duncan's multiple range tests as reported by Gomez [14]. Statistical analysis was performed with SAS computer software. Based on average of two seasons, the results of total yield were used to calculate the costs, benefits and saving of using grafted and non-grafted cantaloupe plants which grown under salinity irrigation water.

Table 1: Effect of salinity levels (dS/m), cultivars and rootstocks as well as their interaction on fruit shape index of cantaloupe plants during 2015 and 2016 seasons.

\begin{tabular}{|c|c|c|c|c|c|c|c|c|c|c|c|}
\hline \multirow{2}{*}{ cv. } & \multirow{2}{*}{ Rootstock } & \multicolumn{5}{|c|}{ First season (2015) } & \multicolumn{5}{|c|}{ Second season (2016) } \\
\hline & & Control & $3.9 \mathrm{dS} / \mathrm{m}$ & $7 \mathrm{dS} / \mathrm{m}$ & $10 \mathrm{dS} / \mathrm{m}$ & Mean & Control & $3.9 \mathrm{dS} / \mathrm{m}$ & $7 \mathrm{dS} / \mathrm{m}$ & $10 \mathrm{dS} / \mathrm{m}$ & Mean \\
\hline \multirow[t]{4}{*}{ Veleta } & Cobalt & $0.98 \mathrm{~A}-\mathrm{E}$ & $0.95 \mathrm{DEF}$ & $0.98 \mathrm{~A}-\mathrm{E}$ & $0.97 \mathrm{~B}-\mathrm{E}$ & $0.97 \mathrm{~b}$ & $0.97 \mathrm{~A}-\mathrm{D}$ & $0.95 \mathrm{~A}-\mathrm{E}$ & $0.96 \mathrm{~A}-\mathrm{D}$ & $0.96 \mathrm{~A}-\mathrm{E}$ & $0.96 \mathrm{a}$ \\
\hline & Strong-Tosa & 1.0 A-D & $0.99 \mathrm{~A}-\mathrm{E}$ & $1.0 \mathrm{~A}-\mathrm{D}$ & $1.04 \mathrm{~A}$ & $1.0 \mathrm{a}$ & $0.96 \mathrm{~A}-\mathrm{D}$ & $0.97 \mathrm{~A}-\mathrm{D}$ & $0.96 \mathrm{~A}-\mathrm{E}$ & $1.00 \mathrm{~A}$ & $0.97 \mathrm{a}$ \\
\hline & $\begin{array}{l}\text { Non- } \\
\text { grafted }\end{array}$ & $1.01 \mathrm{~A}-\mathrm{D}$ & $0.97 \mathrm{~B}-\mathrm{E}$ & $1.02 \mathrm{ABC}$ & $1.03 \mathrm{AB}$ & $1.0 \mathrm{a}$ & $0.97 \mathrm{~A}-\mathrm{D}$ & $0.98 \mathrm{ABC}$ & $0.99 \mathrm{AB}$ & $1.00 \mathrm{~A}$ & $0.99 \mathrm{a}$ \\
\hline & Mean & $0.99 \mathrm{ab}$ & $0.97 \mathrm{bc}$ & $1.0 \mathrm{ab}$ & $1.0 \mathrm{a}$ & & $0.97 \mathrm{a}$ & $0.96 \mathrm{a}$ & $0.97 \mathrm{a}$ & $0.99 \mathrm{a}$ & \\
\hline \multicolumn{2}{|c|}{ Mean Veleta } & \multicolumn{5}{|c|}{$0.99 \mathrm{~A}$} & \multicolumn{5}{|c|}{$0.97 \mathrm{~A}$} \\
\hline \multirow[t]{4}{*}{ Ideal } & Cobalt & $0.94 \mathrm{DEF}$ & $0.94 \mathrm{DEF}$ & $0.93 \mathrm{EF}$ & $0.90 \mathrm{~F}$ & $0.93 \mathrm{c}$ & $0.92 \mathrm{~B}-\mathrm{F}$ & $0.92 \mathrm{~B}-\mathrm{F}$ & $0.93 \mathrm{~A}-\mathrm{E}$ & $0.89 \mathrm{EF}$ & 0.92 bc \\
\hline & Strong-Tosa & $0.92 \mathrm{EF}$ & $0.90 \mathrm{~F}$ & $0.90 \mathrm{~F}$ & $0.90 \mathrm{~F}$ & $0.90 \mathrm{c}$ & $0.93 \mathrm{~A}-\mathrm{E}$ & $0.89 \mathrm{EF}$ & $0.90 \mathrm{DEF}$ & $0.85 \mathrm{~F}$ & $0.89 \mathrm{c}$ \\
\hline & $\begin{array}{c}\text { Non- } \\
\text { grafted }\end{array}$ & $0.95 \mathrm{C}-\mathrm{F}$ & $0.90 \mathrm{~F}$ & $0.93 \mathrm{EF}$ & $0.92 \mathrm{EF}$ & $0.93 \mathrm{c}$ & $0.94 \mathrm{~A}-\mathrm{E}$ & $0.94 \mathrm{~A}-\mathrm{E}$ & $0.91 \mathrm{C}-\mathrm{F}$ & $0.93 \mathrm{~A}-\mathrm{E}$ & $0.93 \mathrm{~b}$ \\
\hline & Mean & $0.94 \mathrm{~cd}$ & $0.91 \mathrm{~d}$ & $0.92 \mathrm{~d}$ & $0.91 \mathrm{~d}$ & & $0.93 \mathrm{~b}$ & $0.91 \mathrm{cb}$ & $0.91 \mathrm{cb}$ & $0.89 \mathrm{c}$ & \\
\hline \multicolumn{2}{|c|}{ Mean Ideal } & \multicolumn{5}{|c|}{$0.92 \mathrm{~B}$} & \multicolumn{5}{|c|}{$0.91 \mathrm{~B}$} \\
\hline \multicolumn{12}{|c|}{ Rootstocks and Salinity levels } \\
\hline \multicolumn{2}{|c|}{ Cobalt } & $0.96 \mathrm{a}$ & $0.94 \mathrm{a}$ & $0.95 \mathrm{a}$ & $0.94 \mathrm{a}$ & $0.95 \mathrm{~A}$ & $0.94 \mathrm{a}$ & $0.93 \mathrm{a}$ & $0.94 \mathrm{a}$ & $0.92 \mathrm{a}$ & $0.96 \mathrm{~A}$ \\
\hline \multicolumn{2}{|c|}{ Strong-Tosa } & $0.96 \mathrm{a}$ & $0.94 \mathrm{a}$ & $0.95 \mathrm{a}$ & $0.97 \mathrm{a}$ & $0.95 \mathrm{~A}$ & $0.95 \mathrm{a}$ & $0.93 \mathrm{a}$ & $0.93 \mathrm{a}$ & $0.93 \mathrm{a}$ & $0.93 \mathrm{~A}$ \\
\hline \multicolumn{2}{|c|}{ Non- grafted } & $0.98 \mathrm{a}$ & $0.93 \mathrm{a}$ & $0.97 \mathrm{a}$ & $0.98 \mathrm{a}$ & $0.97 \mathrm{~A}$ & $0.95 \mathrm{a}$ & $0.96 \mathrm{a}$ & $0.95 \mathrm{a}$ & $0.97 \mathrm{a}$ & $0.96 \mathrm{~A}$ \\
\hline \multicolumn{2}{|c|}{ Mean salinity } & $0.97 \mathrm{~A}$ & $0.94 \mathrm{~A}$ & $0.96 \mathrm{~A}$ & $0.96 \mathrm{~A}$ & & $0.95 \mathrm{~A}$ & $0.94 \mathrm{~A}$ & $0.94 \mathrm{~A}$ & $0.94 \mathrm{~A}$ & \\
\hline
\end{tabular}

\section{Results and Discussion}

Effect of grafting technique (cultivars "scions" and rootstocks) under salinity levels of irrigation water on quality of cantaloupe fruits

Data presented in Tables 1-5 indicate the effect of salinity levels of irrigation water, cultivars, rootstocks and their interaction on fruit shape index, average fruit weight, flesh thickness of fruit, seed cavity diameter and T.S.S, respectively. Fruits quality expressed as average fruit weight, flesh thickness of fruit and T.S.S. were affected by salinity levels of irrigation water but fruit shape index was not affected in both seasons of study. Where, average fruit weight and flesh thickness of fruit were decreased by increasing salinity levels
$[15,16]$ and the opposite trend was observed with T.S.S which increased by increasing salinity levels $[16,17]$. Concerning the effect of cultivars on these traits of fruits quality, all fruit traits except T.S.S. were significantly affected by the used cultivars (Veleta and Ideal). In general, Ideal cultivar fruits were bigger and heavier than those of cv. Veleta. While Veleta fruits were the longer little than those of Ideal and the opposite trend at the fruit diameter all over the growing season, this reflected on the fruit shape index where cv. Veleta recorded the highest value compared with cv. Ideal. Moreover, results indicate that average fruit weight, flesh thickness and seed cavity diameter were positively affected by Cobalt rootstock while no significant effect could be detected regarding to fruit shape 
index and T.S.S. The obtained results agreed with those stated by cucumber who noticed that grafted plants produced fruits with Colla [9] working on watermelon, [18] and Colla [8] working on highest average weight compared with of non-grafted plants.

Table 2: Effect of salinity levels (dS/m), cultivars and rootstocks as well as their interaction on average fruit weight (g) of cantaloupe plants during 2015 and 2016 seasons.

\begin{tabular}{|c|c|c|c|c|c|c|c|c|c|c|c|}
\hline \multirow{2}{*}{ cv. } & \multirow{2}{*}{ Rootstock } & \multicolumn{5}{|c|}{ First season (2015) } & \multicolumn{5}{|c|}{ Second season (2016) } \\
\hline & & Control & $3.9 \mathrm{dS} / \mathrm{m}$ & $7 \mathrm{dS} / \mathrm{m}$ & $10 \mathrm{dS} / \mathrm{m}$ & Mean & Control & $3.9 \mathrm{dS} / \mathrm{m}$ & $7 \mathrm{dS} / \mathrm{m}$ & $10 \mathrm{dS} / \mathrm{m}$ & Mean \\
\hline \multirow[t]{4}{*}{ Veleta } & Cobalt & $1255 \mathrm{CDE}$ & $1314 \mathrm{BC}$ & $1249 \mathrm{CDE}$ & $1044 \mathrm{HI}$ & $1215 \mathrm{ab}$ & $1255 \mathrm{CDE}$ & $1294 \mathrm{~B}$ & 1261B & $973 \mathrm{FG}$ & $1202 \mathrm{~b}$ \\
\hline & $\begin{array}{c}\text { Strong- } \\
\text { Tosa }\end{array}$ & $1167 \mathrm{EFG}$ & 997 IJ & $895 \mathrm{~J}$ & $621 \mathrm{~L}$ & $920 \mathrm{c}$ & $1032 \mathrm{EFG}$ & $1074 \mathrm{DEF}$ & $943 \mathrm{G}$ & $707 \mathrm{H}$ & $939 c$ \\
\hline & $\begin{array}{l}\text { Non- } \\
\text { grafted }\end{array}$ & $1200 \mathrm{DEF}$ & $1020 \mathrm{I}$ & $901 \mathrm{~J}$ & $606 \mathrm{~L}$ & $932 \mathrm{c}$ & $1131 \mathrm{CDE}$ & $1131 \mathrm{CDE}$ & $1003 \mathrm{FG}$ & $702 \mathrm{H}$ & $992 \mathrm{c}$ \\
\hline & Mean & $1207 \mathrm{ab}$ & 1109 bc & $1015 \mathrm{~cd}$ & $757 \mathrm{e}$ & & 1147 c & $1166 \mathrm{bc}$ & $1069 \mathrm{c}$ & $794 \mathrm{~d}$ & \\
\hline \multicolumn{2}{|c|}{ Mean Veleta } & \multicolumn{5}{|c|}{$1022 \mathrm{~B}$} & \multicolumn{5}{|c|}{$1044 \mathrm{~B}$} \\
\hline \multirow[t]{4}{*}{ Ideal } & Cobalt & $1299 \mathrm{BCD}$ & $1312 \mathrm{BC}$ & $1263 \mathrm{CDE}$ & 1004 I & $1220 \mathrm{ab}$ & 1296 B & 1149 CD & $1299 \mathrm{~B}$ & 1004 I & $1173 \mathrm{~b}$ \\
\hline & $\begin{array}{c}\text { Strong- } \\
\text { Tosa }\end{array}$ & $1421 \mathrm{~A}$ & $1463 \mathrm{~A}$ & $1392 \mathrm{AB}$ & $1094 \mathrm{GHI}$ & 1342 a & $1496 \mathrm{~A}$ & $1523 \mathrm{~A}$ & $1478 \mathrm{~B}$ & $950 \mathrm{G}$ & $1381 \mathrm{a}$ \\
\hline & $\begin{array}{l}\text { Non- } \\
\text { grafted }\end{array}$ & $1254 \mathrm{CDE}$ & $1272 \mathrm{CDE}$ & $1131 \mathrm{FGH}$ & $768 \mathrm{~K}$ & $1106 \mathrm{~b}$ & 1215 BC CDE & $1218 \mathrm{BC}$ & $1143 \mathrm{CDE}$ & $774 \mathrm{H}$ & $1087 \mathrm{bc}$ \\
\hline & Mean & $1324 \mathrm{a}$ & 1349 a & $1262 \mathrm{a}$ & $955 \mathrm{~d}$ & & $1335 \mathrm{a}$ & $1296 \mathrm{ab}$ & $1306 \mathrm{ab}$ & $917 \mathrm{~d}$ & \\
\hline \multicolumn{2}{|c|}{ Mean Ideal } & \multicolumn{5}{|c|}{$1223 \mathrm{~A}$} & \multicolumn{5}{|c|}{$1214 \mathrm{~A}$} \\
\hline \multicolumn{12}{|c|}{ Rootstocks and Salinity levels } \\
\hline \multicolumn{2}{|c|}{ Cobalt } & 1217 a & 1313 a & $1256 \mathrm{a}$ & 1024 bc & $1217 \mathrm{~A}$ & $1287 \mathrm{a}$ & $1221 \mathrm{ab}$ & $1280 \mathrm{ab}$ & $961 \mathrm{~cd}$ & $1188 \mathrm{~A}$ \\
\hline \multicolumn{2}{|c|}{ Strong-Tosa } & $1294 \mathrm{a}$ & 1229 a & $1146 \mathrm{ab}$ & $857 \mathrm{~cd}$ & 1131 B & $1264 \mathrm{ab}$ & 1299 a & $1210 \mathrm{ab}$ & $868 \mathrm{de}$ & $1144 \mathrm{~B}$ \\
\hline \multicolumn{2}{|c|}{ Non- grafted } & 1227 a & $1143 \mathrm{ab}$ & $1016 \mathrm{bc}$ & $687 \mathrm{~d}$ & $1019 \mathrm{C}$ & $1173 \mathrm{ab}$ & $1174 \mathrm{ab}$ & 1073 bc & 738 e & $1039 \mathrm{C}$ \\
\hline \multicolumn{2}{|c|}{ Mean salinity } & $1266 \mathrm{~A}$ & $1229 \mathrm{~A}$ & $1138 \mathrm{~B}$ & $856 \mathrm{C}$ & & $1220 \mathrm{~A}$ & $1231 \mathrm{~A}$ & $1187 \mathrm{~B}$ & $856 \mathrm{C}$ & \\
\hline
\end{tabular}

Table 3: Effect of salinity levels (dS/m), cultivars and rootstocks as well as their interaction on flesh thickness (cm) of cantaloupe fruit during 2015 and 2016 seasons.

\begin{tabular}{|c|c|c|c|c|c|c|c|c|c|c|c|}
\hline \multirow{2}{*}{ cv. } & \multirow{2}{*}{ Rootstock } & \multicolumn{5}{|c|}{ First season (2015) } & \multicolumn{5}{|c|}{ Second season (2016) } \\
\hline & & Control & $3.9 \mathrm{dS} / \mathrm{m}$ & $7 \mathrm{dS} / \mathrm{m}$ & $10 \mathrm{dS} / \mathrm{m}$ & Mean & Control & $3.9 \mathrm{dS} / \mathrm{m}$ & $7 \mathrm{dS} / \mathrm{m}$ & $10 \mathrm{dS} / \mathrm{m}$ & Mean \\
\hline \multirow[t]{4}{*}{ Veleta } & Cobalt & $4.0 \mathrm{CD}$ & $4.1 \mathrm{C}$ & 3.7 EFG & $2.9 \mathrm{JK}$ & $3.7 \mathrm{ab}$ & $3.9 \mathrm{CD}$ & $4.1 \mathrm{CD}$ & $3.7 \mathrm{E}$ & $2.9 \mathrm{JKL}$ & $3.6 \mathrm{ab}$ \\
\hline & $\begin{array}{c}\text { Strong- } \\
\text { Tosa }\end{array}$ & $2.9 \mathrm{JK}$ & $3.3 \mathrm{HI}$ & $2.8 \mathrm{JK}$ & $2.5 \mathrm{~L}$ & $2.9 \mathrm{~d}$ & $3.3 \mathrm{GHI}$ & $3.3 \mathrm{GH}$ & $3.8 \mathrm{DE}$ & $2.6 \mathrm{~L}$ & $3.1 \mathrm{c}$ \\
\hline & $\begin{array}{c}\text { Non- } \\
\text { grafted }\end{array}$ & $3.5 \mathrm{FGH}$ & 3.6 FGH & $3.0 \mathrm{~J}$ & $2.5 \mathrm{~L}$ & $3.1 \mathrm{~d}$ & $3.3 \mathrm{FG}$ & $3.6 \mathrm{EF}$ & $3.0 \mathrm{IJ}$ & $2.5 \mathrm{~L}$ & $3.1 \mathrm{c}$ \\
\hline & Mean & $3.5 \mathrm{~cd}$ & $3.6 \mathrm{bc}$ & $3.1 \mathrm{de}$ & $2.7 \mathrm{f}$ & & $3.5 \mathrm{~cd}$ & $3.6 \mathrm{bc}$ & $3.2 \mathrm{~d}$ & $2.7 \mathrm{e}$ & \\
\hline \multicolumn{2}{|c|}{ Mean Vleta } & \multicolumn{5}{|c|}{$3.2 \mathrm{~B}$} & \multicolumn{5}{|c|}{$3.1 \mathrm{~B}$} \\
\hline \multirow[t]{4}{*}{ Ideal } & Cobalt & $3.9 \mathrm{CDE}$ & $4.2 \mathrm{BC}$ & $3.4 \mathrm{GH}$ & $2.9 \mathrm{JK}$ & $3.5 \mathrm{bc}$ & $3.9 \mathrm{CD}$ & $4.1 \mathrm{BC}$ & $3.0 \mathrm{IJ}$ & $2.7 \mathrm{KL}$ & $3.5 \mathrm{bc}$ \\
\hline & $\begin{array}{c}\text { Strong- } \\
\text { Tosa }\end{array}$ & $4.4 \mathrm{AB}$ & $4.6 \mathrm{~A}$ & $3.9 \mathrm{CDE}$ & $3.1 \mathrm{IJ}$ & $4.0 \mathrm{a}$ & $4.3 \mathrm{~B}$ & $4.5 \mathrm{~A}$ & $3.8 \mathrm{DE}$ & $2.9 \mathrm{JKL}$ & $3.9 \mathrm{a}$ \\
\hline & $\begin{array}{c}\text { Non- } \\
\text { grafted }\end{array}$ & 3.6 FGH & $3.8 \mathrm{DEF}$ & $30 \mathrm{~J}$ & $2.7 \mathrm{KL}$ & $3.3 \mathrm{~cd}$ & 3.4 FG & $3.6 \mathrm{EF}$ & $3.1 \mathrm{GHI}$ & $2.7 \mathrm{KL}$ & $3.2 \mathrm{c}$ \\
\hline & Mean & $3.9 \mathrm{ab}$ & $4.2 \mathrm{a}$ & $3.4 \mathrm{~cd}$ & $2.9 \mathrm{fe}$ & & $3.9 \mathrm{ab}$ & $4.1 \mathrm{a}$ & $3.3 \mathrm{~cd}$ & $2.8 \mathrm{e}$ & \\
\hline \multicolumn{2}{|c|}{ Mean Ideal } & \multicolumn{5}{|c|}{$3.6 \mathrm{~A}$} & \multicolumn{5}{|c|}{$3.5 \mathrm{~A}$} \\
\hline \multicolumn{12}{|c|}{ Rootstocks and Salinity levels } \\
\hline \multicolumn{2}{|c|}{ Cobalt } & $3.9 \mathrm{ab}$ & $4.1 \mathrm{a}$ & 3.0 de & $2.9 \mathrm{de}$ & $3.6 \mathrm{~A}$ & $3.9 \mathrm{ab}$ & $4.0 \mathrm{a}$ & 3.3 de & $2.9 \mathrm{f}$ & $3.5 \mathrm{~A}$ \\
\hline \multicolumn{2}{|c|}{ Strong-Tosa } & $3.6 \mathrm{abc}$ & $3.9 \mathrm{ab}$ & $3.4 \mathrm{~cd}$ & $2.8 \mathrm{e}$ & $3.4 \mathrm{~B}$ & 3.8 abcc & $3.9 \mathrm{ab}$ & $3.4 \mathrm{~cd}$ & $2.8 \mathrm{f}$ & $3.5 \mathrm{~A}$ \\
\hline \multicolumn{2}{|c|}{ Non-grafted } & $3.5 \mathrm{bc}$ & $3.7 \mathrm{abc}$ & 3.0 de & $2.6 \mathrm{e}$ & $3.2 \mathrm{C}$ & 3.3 de & $3.6 \mathrm{bcd}$ & 3.0 ef & $2.6 \mathrm{f}$ & $3.1 \mathrm{~B}$ \\
\hline \multicolumn{2}{|c|}{ Mean salinity } & $3.7 \mathrm{~B}$ & $3.9 \mathrm{~A}$ & $3.3 \mathrm{C}$ & $2.7 \mathrm{D}$ & & $3.7 \mathrm{~B}$ & $3.9 \mathrm{~A}$ & $3.2 \mathrm{C}$ & $2.7 \mathrm{D}$ & \\
\hline
\end{tabular}


Table 4: Effect of salinity levels $(\mathrm{dS} / \mathrm{m})$, cultivars and rootstocks as well as their interaction on diameter of seed cavity (cm) of cantaloupe fruit during 2015 and 2016 seasons.

\begin{tabular}{|c|c|c|c|c|c|c|c|c|c|c|c|}
\hline \multirow{2}{*}{ cv. } & \multirow{2}{*}{ Rootstock } & \multicolumn{5}{|c|}{ First season (2015) } & \multicolumn{5}{|c|}{ Second season (2016) } \\
\hline & & Control & $3.9 \mathrm{dS} / \mathrm{m}$ & $7 \mathrm{dS} / \mathrm{m}$ & $10 \mathrm{dS} / \mathrm{m}$ & Mean & Control & $3.9 \mathrm{dS} / \mathrm{m}$ & $7 \mathrm{dS} / \mathrm{m}$ & $10 \mathrm{dS} / \mathrm{m}$ & Mean \\
\hline \multirow[t]{4}{*}{ Veleta } & Cobalt & 3.8 DEF & $4.8 \mathrm{ABC}$ & 4.9 ABC & $5.1 \mathrm{ABC}$ & $4.7 \mathrm{a}$ & 4.7 A-E & $5.2 \mathrm{ABC}$ & $5.6 \mathrm{~A}$ & $5.5 \mathrm{~A}$ & $5.2 \mathrm{a}$ \\
\hline & Strong-Tosa & $4.9 \mathrm{ABC}$ & 3.7 DEF & $3.4 \mathrm{FG}$ & $3.0 \mathrm{FG}$ & $3.8 \mathrm{~b}$ & $5.2 \mathrm{ABC}$ & $5.2 \mathrm{ABC}$ & $3.5 \mathrm{E}-\mathrm{H}$ & $3.1 \mathrm{H}$ & $4.3 \mathrm{bc}$ \\
\hline & Non- grafted & $3.6 \mathrm{EF}$ & 3.4 FG & $2.7 \mathrm{GH}$ & $2.1 \mathrm{H}$ & $3.0 \mathrm{c}$ & 5.0 A-D & 3.8 E-H & $4.2 \mathrm{~B}-\mathrm{H}$ & $3.5 \mathrm{E}-\mathrm{H}$ & $4.1 \mathrm{c}$ \\
\hline & Mean & $4.1 \mathrm{~b}$ & $4.0 \mathrm{~b}$ & $3.7 \mathrm{~b}$ & $3.4 \mathrm{~b}$ & & $5.0 \mathrm{ab}$ & $4.7 \mathrm{ab}$ & $4.4 \mathrm{ab}$ & $4.0 \mathrm{~b}$ & \\
\hline \multicolumn{2}{|c|}{ Mean Veleta } & \multicolumn{5}{|c|}{$3.8 \mathrm{~B}$} & \multicolumn{5}{|c|}{$4.5 \mathrm{~A}$} \\
\hline \multirow[t]{4}{*}{ Ideal } & Cobalt & 4.5 BCD & 3.9 DEF & $5.4 \mathrm{AB}$ & 4.4 CDE & $4.5 \mathrm{a}$ & $5.2 \mathrm{ABC}$ & $3.3 \mathrm{GH}$ & $5.5 \mathrm{~A}$ & 4.0 C-H & $4.5 \mathrm{abc}$ \\
\hline & Strong-Tosa & $3.6 \mathrm{EF}$ & 3.8 DEF & 4.6 BCD & $4.9 \mathrm{ABC}$ & $4.2 \mathrm{ab}$ & $4.2 \mathrm{~B}-\mathrm{H}$ & $3.8 \mathrm{E}-\mathrm{H}$ & $4.4 \mathrm{~A}-\mathrm{G}$ & $4.7 \mathrm{~A}-\mathrm{F}$ & $4.3 \mathrm{bc}$ \\
\hline & Non- grafted & 4.4 CDE & $5.1 \mathrm{ABC}$ & $5.7 \mathrm{~A}$ & $3.1 \mathrm{FG}$ & $4.6 \mathrm{a}$ & $5.7 \mathrm{~A}$ & $5.3 \mathrm{AB}$ & $5.6 \mathrm{~A}$ & 3.4 FGH & $5.0 \mathrm{ab}$ \\
\hline & Mean & $4.2 \mathrm{~b}$ & $4.3 \mathrm{~b}$ & $5.2 \mathrm{a}$ & $4.2 \mathrm{~b}$ & & $5.0 \mathrm{ab}$ & $4.1 \mathrm{~b}$ & $5.2 \mathrm{a}$ & $4.1 \mathrm{~b}$ & \\
\hline \multicolumn{2}{|c|}{ Mean Ideal } & \multicolumn{5}{|c|}{$4.5 \mathrm{~A}$} & \multicolumn{5}{|c|}{$4.6 \mathrm{~A}$} \\
\hline \multicolumn{12}{|c|}{ Rootstocks and Salinity levels } \\
\hline \multicolumn{2}{|c|}{ Cobalt } & $4.1 \mathrm{ab}$ & $4.4 \mathrm{ab}$ & $5.2 \mathrm{a}$ & $4.8 \mathrm{ab}$ & $4.6 \mathrm{~A}$ & $4.9 \mathrm{abc}$ & $4.2 \mathrm{bcd}$ & $5.6 \mathrm{a}$ & $4.8 \mathrm{abc}$ & $4.9 \mathrm{~A}$ \\
\hline \multicolumn{2}{|c|}{ Strong-Tosa } & $4.3 \mathrm{ab}$ & $3.8 \mathrm{~b}$ & $4.0 \mathrm{ab}$ & $4.0 \mathrm{ab}$ & $4.0 \mathrm{~B}$ & $4.7 \mathrm{abc}$ & $4.5 \mathrm{a}-\mathrm{d}$ & $4.0 \mathrm{~cd}$ & $3.9 \mathrm{~cd}$ & $4.3 \mathrm{~B}$ \\
\hline \multicolumn{2}{|c|}{ Non-grafted } & $4.0 \mathrm{~b}$ & $4.3 \mathrm{ab}$ & $4.2 \mathrm{ab}$ & $2.6 \mathrm{c}$ & $3.8 \mathrm{~B}$ & $5.4 \mathrm{ab}$ & $4.6 \mathrm{a}-\mathrm{d}$ & $4.9 \mathrm{abc}$ & $3.5 \mathrm{~d}$ & $4.6 \mathrm{~B}$ \\
\hline \multicolumn{2}{|c|}{ Mean salinity } & $4.1 \mathrm{AB}$ & $4.1 \mathrm{AB}$ & $4.4 \mathrm{~A}$ & $3.8 \mathrm{~B}$ & & $5.0 \mathrm{~A}$ & $4.4 \mathrm{~B}$ & $4.8 \mathrm{~A}$ & $4.0 \mathrm{C}$ & \\
\hline
\end{tabular}

Table 5: Effect of salinity levels (dS/m), cultivars and rootstocks as well as their interaction on fruit T.S.S. of cantaloupe plants during 2015 and 2016 seasons.

\begin{tabular}{|c|c|c|c|c|c|c|c|c|c|c|c|}
\hline \multirow{2}{*}{ cv. } & \multirow{2}{*}{ Rootstock } & \multicolumn{5}{|c|}{ First season (2015) } & \multicolumn{5}{|c|}{ Second season (2016) } \\
\hline & & Control & $3.9 \mathrm{dS} / \mathrm{m}$ & $7 \mathrm{dS} / \mathrm{m}$ & $10 \mathrm{dS} / \mathrm{m}$ & Mean & Control & $3.9 \mathrm{dS} / \mathrm{m}$ & $7 \mathrm{dS} / \mathrm{m}$ & $10 \mathrm{dS} / \mathrm{m}$ & Mean \\
\hline \multirow[t]{4}{*}{ Veleta } & Cobalt & 9.3 FGH & $10.5 \mathrm{DEF}$ & $10.8 \mathrm{CDE}$ & $12.0 \mathrm{ABC}$ & $10.7 \mathrm{a}$ & $8.7 \mathrm{HI}$ & $9.9 \mathrm{FG}$ & $11.1 \mathrm{DE}$ & $12.3 \mathrm{ABC}$ & $10.5 \mathrm{a}$ \\
\hline & Strong-Tosa & $9.3 \mathrm{FGH}$ & $9.9 \mathrm{E}-\mathrm{H}$ & $10.8 \mathrm{CDE}$ & $12.3 \mathrm{AB}$ & $10.6 \mathrm{a}$ & $8.4 \mathrm{I}$ & $9.6 \mathrm{FGH}$ & $11.4 \mathrm{CD}$ & $12.3 \mathrm{ABC}$ & $10.4 \mathrm{a}$ \\
\hline & Non- grafted & $8.7 \mathrm{H}$ & $9.0 \mathrm{GH}$ & 9.9 E-H & $12.0 \mathrm{ABC}$ & $9.9 \mathrm{a}$ & $8.7 \mathrm{HI}$ & $9.6 \mathrm{FGH}$ & 11.1 DE & $12.6 \mathrm{AB}$ & $10.5 \mathrm{a}$ \\
\hline & Mean & $9.1 \mathrm{~d}$ & $9.8 \mathrm{~cd}$ & $10.5 \mathrm{bc}$ & $12.1 \mathrm{a}$ & & $8.6 \mathrm{e}$ & $9.7 \mathrm{~d}$ & $11.2 \mathrm{c}$ & $12.4 \mathrm{~b}$ & \\
\hline \multicolumn{2}{|c|}{ Mean Vleta } & \multicolumn{5}{|c|}{$10.4 \mathrm{~A}$} & \multicolumn{5}{|c|}{$10.5 \mathrm{~A}$} \\
\hline \multirow[t]{4}{*}{ Ideal } & Cobalt & $9.0 \mathrm{GH}$ & $9.9 \mathrm{E}-\mathrm{H}$ & $10.8 \mathrm{CDE}$ & $12.3 \mathrm{AB}$ & $10.5 \mathrm{a}$ & $8.7 \mathrm{HI}$ & $9.9 \mathrm{FG}$ & 11.7 BCD & $13.2 \mathrm{~A}$ & $10.9 \mathrm{a}$ \\
\hline & Strong-Tosa & $9.6 \mathrm{E}-\mathrm{H}$ & $10.2 \mathrm{D}-\mathrm{G}$ & 11.4 BCD & $13.2 \mathrm{~A}$ & $11.1 \mathrm{a}$ & $9.0 \mathrm{GHI}$ & $10.2 \mathrm{EF}$ & $11.4 \mathrm{CD}$ & $13.2 \mathrm{~A}$ & $11.0 \mathrm{a}$ \\
\hline & Non- grafted & $8.7 \mathrm{H}$ & $9.6 \mathrm{E}-\mathrm{H}$ & $10.2 \mathrm{D}-\mathrm{G}$ & $12.3 \mathrm{AB}$ & $10.2 \mathrm{a}$ & $8.4 \mathrm{I}$ & $10.2 \mathrm{EF}$ & $11.1 \mathrm{DE}$ & $12.6 \mathrm{AB}$ & $10.6 \mathrm{a}$ \\
\hline & Mean & $9.1 \mathrm{~d}$ & $9.9 \mathrm{c}$ & $10.8 \mathrm{~b}$ & $12.6 \mathrm{a}$ & & 8.7 e & $10.1 \mathrm{~d}$ & $11.4 \mathrm{c}$ & $13.0 \mathrm{a}$ & \\
\hline \multicolumn{2}{|c|}{ Mean Ideal } & \multicolumn{5}{|c|}{$10.6 \mathrm{~A}$} & \multicolumn{5}{|c|}{$10.8 \mathrm{~A}$} \\
\hline \multicolumn{12}{|c|}{ Rootstocks and Salinity levels } \\
\hline \multicolumn{2}{|c|}{ Cobalt } & $9.2 \mathrm{f}$ & $10.2 \mathrm{~cd}$ & $10.8 \mathrm{bc}$ & $12.2 \mathrm{a}$ & $10.6 \mathrm{~A}$ & $8.7 \mathrm{~d}$ & $9.9 \mathrm{c}$ & $11.4 \mathrm{~b}$ & $12.8 \mathrm{a}$ & $10.7 \mathrm{~A}$ \\
\hline \multicolumn{2}{|c|}{ Strong-Tosa } & 9.5 def & $10.1 \mathrm{cde}$ & $11.1 \mathrm{~b}$ & $12.8 \mathrm{a}$ & $10.8 \mathrm{~A}$ & $8.7 \mathrm{~d}$ & $9.9 \mathrm{c}$ & $11.4 \mathrm{~b}$ & $12.8 \mathrm{a}$ & $10.7 \mathrm{~A}$ \\
\hline \multicolumn{2}{|c|}{ Non- grafted } & $8.7 \mathrm{f}$ & 9.3 ef & 10.1 cde & $12.2 \mathrm{a}$ & $10.1 \mathrm{~A}$ & $8.6 \mathrm{~d}$ & $9.9 \mathrm{c}$ & $11.1 \mathrm{~b}$ & $12.6 \mathrm{a}$ & $10.5 \mathrm{~A}$ \\
\hline \multicolumn{2}{|c|}{ Mean salinity } & $9.1 \mathrm{D}$ & $9.9 \mathrm{C}$ & $10.7 \mathrm{~B}$ & $12.4 \mathrm{~A}$ & & $8.7 \mathrm{D}$ & $9.9 \mathrm{C}$ & $11.3 \mathrm{~B}$ & $12.7 \mathrm{~A}$ & \\
\hline
\end{tabular}

Most fruit quality parameters, i.e., fruit shape index, average fruit weight and T.S.S. were not significantly affected by various trials of the interaction between cultivars and salinity levels and the opposite trend was found with flesh thickness and seed cavity diameter in 2015 and 2016 seasons. However, average fruit weight, flesh thickness and seed cavity diameter as well as T.S.S were significantly affected by the interaction between rootstocks and salinity levels treatments where the highest values were represented in Cobalt rootstock when irrigated by salinity levels 3.9 and $7 \mathrm{dS} / \mathrm{m}$ but increasing salinity up to $10 \mathrm{dS} / \mathrm{m}$ improved T.S.S. Meanwhile fruit shape index was not affected by this interaction. In connection with the interaction treatments between cultivars "scions" and rootstocks, the average fruit weight, flesh thickness and seed cavity diameter were positively significant affected by the grafting combinations of Veleta/Cobalt, Ideal/Cobalt and Ideal/Strong-Tosa compared with non-grafted plants (control) but T.S.S and fruit shape index were not affected by various trials of grafted plants in two seasons. Regarding to the effect of the 
interaction treatments among salinity levels of irrigation water, cultivars and rootstocks, there were significant interaction effects on all fruits quality parameters except fruit shape index. Under all studied factors, the best interaction effects were found in the combination of the cvs. Veleta or Ideal grafted on Cobalt rootstock under all salinity levels except the highest one $(10 \mathrm{dS} / \mathrm{m})$. Where, grafted plants of Ideal/Strong-Tosa, Veleta/Cobalt and Ideal/Cobalt produced the biggest and heaviest fruits with the biggest flesh thickness in a suitable contained of T.S.S when irrigated by $3.9 \mathrm{dS} / \mathrm{m}$ level of salinity levels in both seasons.

Table 6: Effect of salinity levels (dS/m), cultivars and rootstocks as well as their interaction on fruit number/plant of cantaloupe plants during 2015 and 2016 seasons.

\begin{tabular}{|c|c|c|c|c|c|c|c|c|c|c|c|}
\hline \multirow{2}{*}{ cv. } & \multirow{2}{*}{ Rootstock } & \multicolumn{5}{|c|}{ First season (2015) } & \multicolumn{5}{|c|}{ Second season (2016) } \\
\hline & & Control & $3.9 \mathrm{dS} / \mathrm{m}$ & $7 \mathrm{dS} / \mathrm{m}$ & $10 \mathrm{dS} / \mathrm{m}$ & Mean & Control & $3.9 \mathrm{dS} / \mathrm{m}$ & $7 \mathrm{dS} / \mathrm{m}$ & $10 \mathrm{dS} / \mathrm{m}$ & Mean \\
\hline \multirow[t]{4}{*}{ Vleta } & Cobalt & $4.0 \mathrm{AB}$ & $4.0 \mathrm{AB}$ & $3.3 \mathrm{BC}$ & $3.3 \mathrm{BC}$ & $3.7 \mathrm{a}$ & $4.0 \mathrm{AB}$ & $4.0 \mathrm{AB}$ & $3.7 \mathrm{ABC}$ & $3.0 \mathrm{CD}$ & $3.7 \mathrm{ab}$ \\
\hline & $\begin{array}{l}\text { Strong- } \\
\text { Tosa }\end{array}$ & $1.3 \mathrm{D}$ & $1.3 \mathrm{D}$ & $1.0 \mathrm{D}$ & $1.0 \mathrm{D}$ & $1.2 \mathrm{c}$ & $1.3 \mathrm{E}$ & $1.0 \mathrm{E}$ & $1.0 \mathrm{E}$ & $1.0 \mathrm{E}$ & $1.1 \mathrm{~d}$ \\
\hline & $\begin{array}{l}\text { Non- } \\
\text { grafted }\end{array}$ & $3.0 \mathrm{BC}$ & $3.3 \mathrm{BC}$ & $2.7 \mathrm{C}$ & $2.7 \mathrm{C}$ & $2.9 \mathrm{~b}$ & 3.3 BCD & $3.3 \mathrm{BCD}$ & $3.0 \mathrm{CD}$ & $2.7 \mathrm{D}$ & $3.1 \mathrm{c}$ \\
\hline & Mean & $2.7 \mathrm{ab}$ & $2.9 \mathrm{ab}$ & $2.3 \mathrm{~b}$ & $2.3 \mathrm{~b}$ & & $2.8 \mathrm{abc}$ & $2.7 \mathrm{abc}$ & $2.5 \mathrm{bc}$ & $2.2 \mathrm{c}$ & \\
\hline \multicolumn{2}{|c|}{ Mean Vleta } & \multicolumn{5}{|c|}{$2.6 \mathrm{~B}$} & \multicolumn{5}{|c|}{$2.6 \mathrm{~B}$} \\
\hline \multirow[t]{4}{*}{ Ideal } & Cobalt & $3.0 \mathrm{BC}$ & $3.3 \mathrm{BC}$ & $3.7 \mathrm{ABC}$ & $\begin{array}{c}233.33 \\
\text { CEF }\end{array}$ & $3.2 \mathrm{~b}$ & 3.3 BCD & $3.7 \mathrm{ABC}$ & $3.3 \mathrm{BCD}$ & $3.0 \mathrm{CD}$ & $3.3 \mathrm{bc}$ \\
\hline & $\begin{array}{l}\text { Strong- } \\
\text { Tosa }\end{array}$ & $3.7 \mathrm{ABC}$ & $4.3 \mathrm{~A}$ & $3.7 \mathrm{ABC}$ & $3.3 \mathrm{BC}$ & 3.7 a & $4.0 \mathrm{~A}$ & $4.3 \mathrm{~A}$ & $3.3 \mathrm{BCD}$ & $3.3 \mathrm{BCD}$ & $3.7 \mathrm{a}$ \\
\hline & $\begin{array}{c}\text { Non- } \\
\text { grafted }\end{array}$ & $2.7 \mathrm{C}$ & $2.7 \mathrm{C}$ & $2.7 \mathrm{C}$ & 190 GHI & $2.8 \mathrm{~b}$ & $3.0 \mathrm{CD}$ & $3.3 \mathrm{BCD}$ & $2.7 \mathrm{D}$ & $2.7 \mathrm{D}$ & $2.9 \mathrm{c}$ \\
\hline & Mean & $3.1 \mathrm{ab}$ & $3.5 \mathrm{a}$ & $3.4 \mathrm{a}$ & $2.9 \mathrm{ab}$ & & $3.4 \mathrm{ab}$ & $3.7 \mathrm{a}$ & $3.1 \mathrm{abc}$ & $3.0 \mathrm{abc}$ & \\
\hline \multicolumn{2}{|c|}{ Mean Ideal } & $3.2 \mathrm{~A}$ & & & & & $3.3 \mathrm{~A}$ & & & & \\
\hline \multicolumn{12}{|c|}{ Rootstocks and Salinity levels } \\
\hline \multicolumn{2}{|c|}{ Cobalt } & $3.5 \mathrm{ab}$ & $3.7 \mathrm{a}$ & $3.5 \mathrm{ab}$ & $3.0 \mathrm{abc}$ & $3.4 \mathrm{~A}$ & $3.6 \mathrm{a}$ & $3.8 \mathrm{a}$ & $3.5 \mathrm{ab}$ & $3.0 \mathrm{ab}$ & $3.5 \mathrm{~A}$ \\
\hline \multicolumn{2}{|c|}{ Strong-Tosa } & $2.5 \mathrm{abc}$ & $2.8 \mathrm{abc}$ & $2.3 \mathrm{bc}$ & $2.1 \mathrm{c}$ & $2.4 \mathrm{C}$ & $2.7 \mathrm{ab}$ & $2.6 \mathrm{ab}$ & $2.2 \mathrm{~b}$ & $2.1 \mathrm{~b}$ & $2.4 \mathrm{C}$ \\
\hline \multicolumn{2}{|c|}{ Non- grafted } & $2.8 \mathrm{abc}$ & $3.2 \mathrm{abc}$ & $2.8 \mathrm{abc}$ & $2.6 \mathrm{abc}$ & $2.8 \mathrm{~B}$ & $3.1 \mathrm{ab}$ & $3.3 \mathrm{ab}$ & $2.8 \mathrm{ab}$ & $2.6 \mathrm{ab}$ & $3.0 \mathrm{~B}$ \\
\hline \multicolumn{2}{|c|}{ Mean salinity } & $2.9 \mathrm{~B}$ & $3.2 \mathrm{~A}$ & $2.8 \mathrm{~B}$ & $2.6 \mathrm{C}$ & & $3.2 \mathrm{AB}$ & $3.3 \mathrm{~A}$ & $2.8 \mathrm{BC}$ & $2.6 \mathrm{C}$ & \\
\hline
\end{tabular}

Table 7: Effect of salinity levels (dS/m), cultivars and rootstocks as well as their interaction on early yield (g / plant) of cantaloupe plants during 2015 and 2016 seasons.

\begin{tabular}{|c|c|c|c|c|c|c|c|c|c|c|c|}
\hline \multirow{2}{*}{ cv. } & \multirow{2}{*}{ Rootstock } & \multicolumn{5}{|c|}{ First season (2015) } & \multicolumn{5}{|c|}{ Second season (2016) } \\
\hline & & Control & $3.9 \mathrm{dS} / \mathrm{m}$ & $7 \mathrm{dS} / \mathrm{m}$ & $10 \mathrm{dS} / \mathrm{m}$ & Mean & Control & $3.9 \mathrm{dS} / \mathrm{m}$ & $7 \mathrm{dS} / \mathrm{m}$ & $10 \mathrm{dS} / \mathrm{m}$ & Mean \\
\hline \multirow[t]{4}{*}{ Veleta } & Cobalt & $1149 \mathrm{JK}$ & $1299 \mathrm{EFG}$ & $1644 \mathrm{~A}$ & $1394 \mathrm{BCD}$ & $1371 \mathrm{a}$ & $1281 \mathrm{~B}$ & $1283 \mathrm{~B}$ & $1274 \mathrm{~B}$ & $1016 \mathrm{FG}$ & 1213ab \\
\hline & Strong-Tosa & $1144 \mathrm{JK}$ & 3070 & 12:00 PM & 12:00 PM & $363 c$ & $323 \mathrm{~L}$ & $0 \mathrm{M}$ & $0 \mathrm{M}$ & $0 \mathrm{M}$ & $81 \mathrm{~d}$ \\
\hline & Non- grafted & $1176 \mathrm{~J}$ & $1333 \mathrm{D}-\mathrm{G}$ & $1185 \mathrm{IJ}$ & $650 \mathrm{~N}$ & $1086 \mathrm{~b}$ & $1135 \mathrm{DE}$ & $1148 \mathrm{D}$ & $665 \mathrm{~K}$ & $718 \mathrm{JK}$ & $916 \mathrm{c}$ \\
\hline & Mean & $1156 \mathrm{a}$ & $980 \mathrm{ab}$ & $943 \mathrm{ab}$ & $681 \mathrm{~b}$ & & $913 \mathrm{abc}$ & $810 \mathrm{bc}$ & $646 \mathrm{bc}$ & $578 \mathrm{c}$ & \\
\hline \multicolumn{2}{|c|}{ Mean Vleta } & \multicolumn{5}{|c|}{$940 \mathrm{~B}$} & \multicolumn{5}{|c|}{$737 \mathrm{~B}$} \\
\hline \multirow[t]{4}{*}{ Ideal } & Cobalt & $1273 \mathrm{GH}$ & $1300 \mathrm{EFG}$ & $1259 \mathrm{GHI}$ & $994 \mathrm{~L}$ & $1207 a b$ & $856 \mathrm{HI}$ & $1161 \mathrm{CD}$ & $1263 \mathrm{BC}$ & $942 \mathrm{GH}$ & $1055 b c$ \\
\hline & Strong-Tosa & $1361 \mathrm{C}-\mathrm{F}$ & $1449 \mathrm{~B}$ & 1377 B-E & $1426 \mathrm{BC}$ & $1403 a$ & $1007 \mathrm{FG}$ & $1517 \mathrm{~A}$ & $1493 \mathrm{~A}$ & $1047 \mathrm{EF}$ & 1266 a \\
\hline & Non- grafted & $1205 \mathrm{IJ}$ & 1286 FGH & $1093 \mathrm{~K}$ & $760 \mathrm{M}$ & $1086 \mathrm{~b}$ & $1203 \mathrm{BCD}$ & $1230 \mathrm{BCD}$ & $1154 \mathrm{D}$ & $802 \mathrm{IJ}$ & $1097 \mathrm{~b}$ \\
\hline & Mean & 1280 a & $1345 \mathrm{a}$ & $1243 \mathrm{a}$ & $1060 \mathrm{ab}$ & & $913 \mathrm{abc}$ & $1302 \mathrm{a}$ & $1304 \mathrm{a}$ & $931 \mathrm{abc}$ & \\
\hline \multicolumn{2}{|c|}{ Mean Ideal } & \multicolumn{5}{|c|}{$1232 \mathrm{~A}$} & \multicolumn{5}{|c|}{$1140 \mathrm{~A}$} \\
\hline \multicolumn{12}{|c|}{ Rootstocks and Salinity levels } \\
\hline & Cobalt & $1211 \mathrm{ab}$ & $1210 \mathrm{ab}$ & $1452 \mathrm{a}$ & $1194 \mathrm{abc}$ & $1289 \mathrm{~A}$ & $1069 \mathrm{abc}$ & $1222 \mathrm{a}$ & $1269 \mathrm{a}$ & $979 \mathrm{abc}$ & $1134 \mathrm{~A}$ \\
\hline & ong-Tosa & $1252 \mathrm{ab}$ & $878 \mathrm{bcd}$ & $689 d$ & $713 \mathrm{~cd}$ & $883 \mathrm{C}$ & $665 \mathrm{bc}$ & $758 \mathrm{abc}$ & $746 \mathrm{abc}$ & $524 \mathrm{c}$ & $673 \mathrm{C}$ \\
\hline & - grafted & $1191 \mathrm{abc}$ & $1309 a b$ & 1139 a-d & $705 \mathrm{~cd}$ & $1086 \mathrm{~B}$ & $1169 \mathrm{ab}$ & $1189 \mathrm{ab}$ & $910 \mathrm{abc}$ & $760 \mathrm{abc}$ & $1007 \mathrm{~B}$ \\
\hline & n salinity & $1218 \mathrm{~A}$ & $1162 \mathrm{~B}$ & $1093 \mathrm{C}$ & $871 \mathrm{D}$ & & $967 \mathrm{~B}$ & $1056 \mathrm{~A}$ & $975 \mathrm{~B}$ & $754 \mathrm{C}$ & \\
\hline
\end{tabular}


Effect of grafting technique (cultivars "scions" and rootstocks) under salinity levels of irrigation water on fruit yield and its components of cantaloupe plants.

Data presented in Tables 6- 8 shows the effect of salinity levels of irrigation water, cultivars, rootstocks, and their interaction on fruits number, early and total yield (g/plant), respectively. Yield production in terms of fruits number, early yield and total yield per plant were significantly affected by salinity levels during both seasons. Where, the medium salinity level $(3.9 \mathrm{dS} / \mathrm{m})$ resulted in significantly the highest of early yield, fruits number and total yield than all other salinity levels while the total yield decreased by $39.7 \%$ (as average between two seasons) with increasing salinity levels up to $10 \mathrm{dS} / \mathrm{m}$. The obtained results are in the same line with those reported by $[3,5,17,19-21]$ who showed that increasing salinity levels badly affected total melon yield. Also, early yield, fruits number and total yield per plant were affected by the used cultivars (Veleta and Ideal) where cv Ideal was higher than those of cv. Veleta. The effect of rootstocks was very clear where Cobalt rootstock produced significantly higher yield components than all other used rootstock and non-grafted plants, increased the total yield by $37.4 \%$ compare non-grafted plants, as average between two seasons. In the same context, [8] on melon as well as [22-26] on watermelon who noticed that grafted plants gave the highest fruit yields compared with non-grafted plants.

Table 8: Effect of salinity levels (dS/m), cultivars and rootstocks as well as their interaction on total fruit yield (g / plant) of cantaloupe plants during 2015 and 2016 seasons.

\begin{tabular}{|c|c|c|c|c|c|c|c|c|c|c|c|}
\hline \multirow{2}{*}{ cv. } & \multirow{2}{*}{ Rootstock } & \multicolumn{5}{|c|}{ First season (2015) } & \multicolumn{5}{|c|}{ Second season (2016) } \\
\hline & & Control & $3.9 \mathrm{dS} / \mathrm{m}$ & $7 \mathrm{dS} / \mathrm{m}$ & $10 \mathrm{dS} / \mathrm{m}$ & Mean & Control & $3.9 \mathrm{dS} / \mathrm{m}$ & $7 \mathrm{dS} / \mathrm{m}$ & $10 \mathrm{dS} / \mathrm{m}$ & Mean \\
\hline \multirow[t]{4}{*}{ Veleta } & Cobalt & $5021 \mathrm{BC}$ & 5249 B & $4165 \mathrm{DEF}$ & $3482 \mathrm{G}$ & 4479ab & $5177 \mathrm{BC}$ & 5177 BC & $4625 \mathrm{CD}$ & 2918 IJ & $4495 \mathrm{~b}$ \\
\hline & Strong-Tosa & $1556 \mathrm{JK}$ & 1329 KL & 894 LM & $621 \mathrm{M}$ & $1100 \mathrm{c}$ & $1367 \mathrm{~L}$ & 1074 LM & 943 LM & $708 \mathrm{M}$ & $1025 \mathrm{e}$ \\
\hline & Non- grafted & $3600 \mathrm{G}$ & $3435 \mathrm{G}$ & $2409 \mathrm{HI}$ & $1636 \mathrm{~J}$ & $2770 \mathrm{c}$ & 3767 FGH & 3782 FGH & 3007 IJ & $1882 \mathrm{~K}$ & $3110 \mathrm{~d}$ \\
\hline & Mean & 3392 bc & 3337 bc & $2489 \mathrm{~cd}$ & $1913 \mathrm{~d}$ & & $3420 \mathrm{~b}$ & $3344 \mathrm{~b}$ & 2858 bc & $1836 \mathrm{c}$ & \\
\hline \multicolumn{2}{|c|}{ Mean Veleta } & \multicolumn{5}{|c|}{$2783 \mathrm{~B}$} & \multicolumn{5}{|c|}{$2865 \mathrm{~B}$} \\
\hline \multirow[t]{4}{*}{ Ideal } & Cobalt & 3896 EFG & $4379 \mathrm{DE}$ & $4630 \mathrm{CD}$ & $2678 \mathrm{H}$ & 3896 b & $4319 \mathrm{DE}$ & $4213 \mathrm{DEF}$ & $4329 \mathrm{DE}$ & $2851 \mathrm{~J}$ & $3928 \mathrm{bc}$ \\
\hline & Strong-Tosa & 5212 B & $6341 \mathrm{~A}$ & $5102 \mathrm{BC}$ & $3647 \mathrm{FG}$ & 5075 a & $5470 \mathrm{AB}$ & $6600 \mathrm{~A}$ & 4928 BC & $3427 \mathrm{HI}$ & $5106 \mathrm{a}$ \\
\hline & Non- grafted & $3331 \mathrm{G}$ & 3815 EFG & $3393 \mathrm{G}$ & $2038 \mathrm{IJ}$ & $3144 \mathrm{c}$ & $3645 \mathrm{GH}$ & $4060 \mathrm{EFG}$ & $3048 \mathrm{IJ}$ & $2063 \mathrm{~K}$ & $3204 \mathrm{~cd}$ \\
\hline & Mean & $4150 \mathrm{ab}$ & $4845 \mathrm{a}$ & $4375 \mathrm{ab}$ & $2787 \mathrm{~cd}$ & & $4334 \mathrm{a}$ & 4958 a & $4102 \mathrm{ab}$ & $2780 \mathrm{bc}$ & \\
\hline \multicolumn{2}{|c|}{ Mean Ideal } & \multicolumn{5}{|c|}{$4039 \mathrm{~A}$} & \multicolumn{5}{|c|}{$4043 \mathrm{~A}$} \\
\hline \multicolumn{12}{|c|}{ Rootstocks and Salinity levels } \\
\hline & Cobalt & $4459 \mathrm{ab}$ & $4814 \mathrm{a}$ & $4397 \mathrm{ab}$ & 3080 a-d & $4187 \mathrm{~A}$ & $4718 \mathrm{a}$ & $4695 \mathrm{a}$ & 4477 a & $2885 \mathrm{ab}$ & $4140 \mathrm{~A}$ \\
\hline & rong-Tosa & 3384 a-d & $3835 \mathrm{abc}$ & 2998 a-d & $2134 \mathrm{~cd}$ & 3088 B & $3423 \mathrm{ab}$ & $3837 \mathrm{ab}$ & $2935 \mathrm{ab}$ & $2067 \mathrm{~b}$ & $3066 \mathrm{~B}$ \\
\hline & n- grafted & 3465 a-d & 3625 a-d & $2901 \mathrm{bcd}$ & $1837 \mathrm{~d}$ & 2958 B & $3706 \mathrm{ab}$ & $3921 \mathrm{ab}$ & $3028 a b$ & $1972 \mathrm{~b}$ & $3157 \mathrm{~B}$ \\
\hline & an salinity & 3772 B & $4091 \mathrm{~A}$ & $3432 \mathrm{C}$ & $2350 \mathrm{D}$ & & $3878 \mathrm{~B}$ & $4151 \mathrm{~A}$ & $3480 \mathrm{C}$ & $2308 \mathrm{D}$ & \\
\hline
\end{tabular}

Yield components except fruits number were significantly affected by the interaction treatments between cultivars and salinity levels, where the highest values were recorded when cv. Ideal was irrigated by salinity level $3.9 \mathrm{dS} / \mathrm{m}$ compared with cv. Veleta which recorded the lowest values at $10 \mathrm{dS} / \mathrm{m}$ level. Early yield and total yield per plant were significantly affected by the interaction treatments between rootstocks and salinity levels while fruits number was not significantly affected by this interaction. As for the early yield, the highest values were recorded by Cobalt rootstock when irrigated by salinity level $7 \mathrm{dS} / \mathrm{m}$ followed by $3.9 \mathrm{dS} / \mathrm{m}$. Meanwhile, the same rootstock resulted in the highest values of fruits number and total yield when irrigated by salinity level 3.9 $\mathrm{dS} / \mathrm{m}$ followed by control level. Yield components were positively affected by graft combinations of Ideal/Strong-Tosa and Veleta/ Cobalt, where the total yield was increased by 60.4 and $52.6 \%$, respectively by these combinations compared with non-grafted of the same cultivar. Meanwhile, non-grafted plants especially Veleta cv. (control) recorded the lowest values of yield components.
The best interaction effects between the three studied factors were found in the combination of Ideal/Strong-Tosa and Veleta/ Cobalt with the lowest $(3.9 \mathrm{dS} / \mathrm{m})$ and control (non-saline) of salinity levels. While non-grafted plants of both cultivars (control) which irrigated by highest salinity level $(10 \mathrm{dS} / \mathrm{m})$ recorded the lowest values. Generally all types of treatments interactions among the three studied factors were significantly higher compared to the control treatment. The interaction between cv. Ideal grafted on Strong-Tosa rootstock as well as Veleta on Cobalt with the nonsaline level (control) and the lowest level $(3.9 \mathrm{dS} / \mathrm{m})$ of salinity levels resulted in the best cantaloupe growth El-S Zaki 2018 and the yield. Whereas graft combination of Ideal/Strong-Tosa increased the total yield by 53.1, 85.5, 43.8 and $1.4 \%$ at salinity levels control, $3.9,7$ and $10 \mathrm{dS} / \mathrm{m}$, respectively compared to the general control (non-grafted plants of Ideal at control water). This percentage was 38.4, 41.5, 19.3 and decline 13.1\% with Veleta/Cobalt compared to the general control (non-grafted plants of Veleta at control water). Generally, when cantaloupe plants have to be irrigated with high 
salinity of irrigation water, it is recommended to cultivate grafted seedling resulted of Veleta/Cobalt and Ideal/Strong-Tosa where these plants resulted the highest benefit and income compared with those on its own roots (non-grafted plants) under each salinity level of irrigation water. Economically, it can be recommend under similar circumstances to use the water with moderate salinity (3.9 and $7 \mathrm{dS} / \mathrm{m}$ ) when combined with grafting seedling (Veleta/ Cobalt and Ideal/Strong-Tosa) in order to get optimum yield with using somewhat saline water.

\section{Calculation of Costs and Benefits of Applied Treatments}

This parameter is illustrative and is not reliable in order to differentiate between the costs of using grafted and non-grafted plants under salinity of irrigation water. Assuming that, the remaining costs such as rental costs, workers, fertilizers, etc., agree on all factors of the study. Costs and benefits of grafted and nongrafted plants which grown under salinity levels of irrigation water were calculated as average between both seasons. Where, the price of non-grafted seedlings is close to the price of the grafted seedlings because it required additional costs "the costs of controlling soil diseases (1.25LE) according to Hasan (2015)". The presented results in Table 9 show the costs (L.E) of the irrigation water was invariable with all salinity levels with average 2.22 L.E. /Plant. Where the highest benefits (10.30 and 9.56 L.E./Plant) and income (8.08 and 7.34 L.E./Plant) were obtained with irrigation by $3.9 \mathrm{dS} / \mathrm{m}$ and non-saline level, respectively. While, the lowest benefits (5.82L.E./ Plant) and income (3.60L.E./Plant) were obtained with cantaloupe plants which irrigated by highest salinity level $(10 \mathrm{dS} / \mathrm{m})$. It is due to increasing the plants yield which irrigated by $3.9 \mathrm{dS} / \mathrm{m}(4.121 \mathrm{~kg} /$ Plant). Using of cv. Ideal obtained the highest benefit (10.10L.E./ Plant) and income (7.83L.E./Plant) compare with those of Veleta plant which recorded the lowest benefit (7.06L.E./Plant) and income (4.89L.E./Plant). It is due to increasing the yield of Ideal cv. (4.042kg./Plant) compare with those of Veleta plants $(2.824 \mathrm{~kg} /$ plant). Also, the highest benefit (10.41L.E./Plant) and income (8.11L.E./Plant) were represented when both cantaloupe cultivars were grafted on rootstock Cobalt although grafted plants recorded the higher costs (2.30L.E/plant) compared with non-grafted plants (2.05 L.E/plant). It is due to increasing the yield of grafted plants on Cobalt rootstock $(4.164 \mathrm{~kg} /$ Plant) compare with those of nongrafted plants $(3.058 \mathrm{~kg} /$ plant).

Table 9: Effect of cultivars and rootstocks under salinity levels on costs and benefits of cantaloupe plants as average between 2015 and 2016 seasons.

\begin{tabular}{|c|c|c|c|c|c|c|c|c|c|c|c|}
\hline \multirow{2}{*}{ cv. } & \multirow{2}{*}{ Rootstock } & \multicolumn{5}{|c|}{ First season (2015) } & \multicolumn{5}{|c|}{ Second season (2016) } \\
\hline & & Control & $3.9 \mathrm{dS} / \mathrm{m}$ & $7 \mathrm{dS} / \mathrm{m}$ & $10 \mathrm{dS} / \mathrm{m}$ & Mean & Control & $3.9 \mathrm{dS} / \mathrm{m}$ & $7 \mathrm{dS} / \mathrm{m}$ & $10 \mathrm{dS} / \mathrm{m}$ & Mean \\
\hline \multirow{4}{*}{ Veleta } & Cobalt & $5021 \mathrm{BC}$ & 5249 B & $4165 \mathrm{DEF}$ & $3482 \mathrm{G}$ & $4479 \mathrm{ab}$ & $5177 \mathrm{BC}$ & $5177 \mathrm{BC}$ & $4625 \mathrm{CD}$ & 2918 IJ & $4495 \mathrm{~b}$ \\
\hline & Strong-Tosa & $1556 \mathrm{JK}$ & $1329 \mathrm{KL}$ & 894 LM & $621 \mathrm{M}$ & $1100 \mathrm{c}$ & $1367 \mathrm{~L}$ & 1074 LM & 943 LM & $708 \mathrm{M}$ & $1025 \mathrm{e}$ \\
\hline & Non- grafted & $3600 \mathrm{G}$ & $3435 \mathrm{G}$ & $2409 \mathrm{HI}$ & $1636 \mathrm{~J}$ & $2770 \mathrm{c}$ & 3767 FGH & $3782 \mathrm{FGH}$ & 3007 IJ & $1882 \mathrm{~K}$ & $3110 \mathrm{~d}$ \\
\hline & Mean & $3392 \mathrm{bc}$ & 3337 bc & $2489 \mathrm{~cd}$ & $1913 \mathrm{~d}$ & & $3420 \mathrm{~b}$ & $3344 \mathrm{~b}$ & 2858 bc & 1836 c & \\
\hline \multicolumn{2}{|c|}{ Mean Veleta } & \multicolumn{5}{|c|}{$2783 \mathrm{~B}$} & \multicolumn{5}{|c|}{$2865 \mathrm{~B}$} \\
\hline \multirow[t]{4}{*}{ Ideal } & Cobalt & $3896 \mathrm{EFG}$ & $4379 \mathrm{DE}$ & $4630 \mathrm{CD}$ & $2678 \mathrm{H}$ & $3896 \mathrm{~b}$ & $4319 \mathrm{DE}$ & $4213 \mathrm{DEF}$ & $4329 \mathrm{DE}$ & $2851 \mathrm{~J}$ & $3928 \mathrm{bc}$ \\
\hline & Strong-Tosa & 5212 B & $6341 \mathrm{~A}$ & $5102 \mathrm{BC}$ & $3647 \mathrm{FG}$ & $5075 \mathrm{a}$ & $5470 \mathrm{AB}$ & $6600 \mathrm{~A}$ & $4928 \mathrm{BC}$ & $3427 \mathrm{HI}$ & $5106 \mathrm{a}$ \\
\hline & Non- grafted & $3331 \mathrm{G}$ & $3815 \mathrm{EFG}$ & $3393 \mathrm{G}$ & $2038 \mathrm{IJ}$ & $3144 \mathrm{c}$ & $3645 \mathrm{GH}$ & $4060 \mathrm{EFG}$ & 3048 IJ & $2063 \mathrm{~K}$ & $3204 \mathrm{~cd}$ \\
\hline & Mean & $4150 \mathrm{ab}$ & $4845 \mathrm{a}$ & $4375 \mathrm{ab}$ & $2787 \mathrm{~cd}$ & & $4334 \mathrm{a}$ & 4958 a & $4102 \mathrm{ab}$ & $2780 \mathrm{bc}$ & \\
\hline \multicolumn{2}{|c|}{ Mean Ideal } & \multicolumn{5}{|c|}{$4039 \mathrm{~A}$} & \multicolumn{5}{|c|}{$4043 \mathrm{~A}$} \\
\hline \multicolumn{12}{|c|}{ Rootstocks and Salinity levels } \\
\hline & obalt & $4459 \mathrm{ab}$ & $4814 \mathrm{a}$ & $4397 \mathrm{ab}$ & 3080 a-d & $4187 \mathrm{~A}$ & $4718 \mathrm{a}$ & $4695 \mathrm{a}$ & 4477 a & $2885 \mathrm{ab}$ & $4140 \mathrm{~A}$ \\
\hline & ng-Tosa & $3384 \mathrm{a}-\mathrm{d}$ & $3835 a b c$ & 2998 a-d & $2134 \mathrm{~cd}$ & $3088 \mathrm{~B}$ & $3423 \mathrm{ab}$ & $3837 \mathrm{ab}$ & $2935 \mathrm{ab}$ & $2067 \mathrm{~b}$ & 3066 B \\
\hline & grafted & 3465 a-d & 3625 a-d & $2901 \mathrm{bcd}$ & $1837 \mathrm{~d}$ & 2958 B & $3706 \mathrm{ab}$ & $3921 \mathrm{ab}$ & $3028 \mathrm{ab}$ & $1972 \mathrm{~b}$ & $3157 \mathrm{~B}$ \\
\hline & salinity & 3772 B & $4091 \mathrm{~A}$ & $3432 \mathrm{C}$ & $2350 \mathrm{D}$ & & 3878 B & $4151 \mathrm{~A}$ & $3480 \mathrm{C}$ & $2308 \mathrm{D}$ & \\
\hline
\end{tabular}

When irrigated the plants by different salinity levels, the Ideal plants recorded the highest benefits (12.25L.E./Plant) and income (9.98L.E./Plant) at 3.9dS/m level, meanwhile the lower benefit (4.69L.E./Plant) and income (2.52L.E./Plant) were resulted with $\mathrm{cv}$. Veleta at the highest salinity level $(10 \mathrm{dS} / \mathrm{m})$. Also under different salinity levels, the highest benefits (11.20, 11.89 and 11.09L.E./Plant) and income (8.90, 9.59 and 8.79L.E./Plant) were represented in rootstock Cobalt and irrigated by non-saline level, $3.9 \mathrm{dS} / \mathrm{m}$ and $7 \mathrm{dS} / \mathrm{m}$ of salinity levels, respectively as compared with non-grafted plants which recorded the lowest benefits $(8.97$,
9.43 and 7.41L.E./Plant) and income (6.92, 7.38 and5.36 L.E./ Plant) at the same salinity levels. This is due to increasing the yield of grafting plants on Cobalt rootstock (4.481, 4.755 and $4.437 \mathrm{~kg}$./ Plant) as well as lower the yield of non-grafted plants (3.590, 3.773 and $2.965 \mathrm{~kg}$./ Plant). The grafting combinations Ideal/Strong-Tosa followed by Veleta/Cobalt then Ideal/Cobalt obtained the highest benefits (12.73, 11.17and 9.65 L.E./Plant) and income (10.38, 8.92 and 7.30 L.E./Plant) while the lowest benefits (2.66, 7.35 and 7.94 L.E./Plant) and income $(0.44,5.35$ and 5.84 L.E./Plant) were obtained by Veleta /Strong-Tosa followed by Veleta and Ideal 
plants on its own roots, respectively. Under all studied factors, the grafting combination Ideal/Strong-Tosa resulted the highest benefit (13.35, 16.18, 12.54 and 8.84L.E./Plant) and income (11.00, 13.83, 10.19 and 6.49L.E./Plant) at non-saline level, $3.9 \mathrm{dS} / \mathrm{m}, 7 \mathrm{dS} / \mathrm{m}$ and $10 \mathrm{dS} / \mathrm{m}$ of salinity, respectively compared with the same $\mathrm{cv}$. on its roots also the graft combination Veleta/Cobalt showed the highest benefit $(12.67,13.03,10.99$ and 8.00L.E./Plant) and income (10.42, 10.78, 8.74 and 5.75 L.E./Plant) at the same salinity levels respectively compared with the same cv. on its roots. This is due to increasing the yield of these grafting plants compared with nongrafted plants of the same cv.

\section{References}

1. Carvalho BL, de IB do Nascimento, J F de Medeiros, SSV de Andrade, JL D Dombroski (2011) Stomatal conductance and leaf area of melon under saline stress in different soils. Green Magazine of Agroecology Sustainable Development 6(2): 1-6.

2. Pereira A de M, RCF de Queiroga, GD da Silva, M das G R do Nascimento, SEO de Andrade (2012) Germination and initial growth of melon seed submitted to the osmopriming with $\mathrm{NaCl}$ and salinity levels of water. Green Magazine on Agroecology and Sustainable Development 7(3): 205-211.

3. Dias N da S, VS Palacio, KKC de F Moura, ON Sousa Neto (2015) Growth of melon plants in coconut saline nutrient solution. IRRIGA 20(1): 1-12.

4. Dias N da S, AM de Oliveira, ON de Sousa Neto, FF Blanco, JRL Reboucas (2011) Salt concentration and phases of exposure to salinity of melon grown in coconut fiber substrate. Brazilian Journal of Fruticulture 33(3): 915-921.

5. Huang CH, L Zong, M Buonanno, X Xue, T Wang, et al. (2012) Impact of saline water irrigation on yield and quality of melon (Cucumis melo cv. Huanghemi) in northwest China. European J Agron 43:68-76.

6. Duarte TS, R MN Peil, S Bacchi (2012) Effect of saline concentrations on growth of melon cultivated under protected environment. Acta Hort 952: 673-678.

7. Estañ MT, MM Martinez Rodriguez, F Perez Alfocea, TJ Flowers, MC Bolarin (2005) Grafting raises the salt tolerance of tomato through limiting the transport of sodium and chloride to the shoot. J Experimental Botany 56(412): 703-712.

8. Colla G, Y Rouphael, M Cardarelli, D Massa, A Salerno, et al. (2006) Yield, fruit quality and mineral composition of grafted melon plants grown under saline conditions. J Hort Sci and Biotech 81(1): 146-152.

9. Colla GY, Roupahel M, Cardarelli, E Rea (2006) Effect of salinity on yield fruit quality, leaf gas exchange, and mineral composition of grafted watermelon plants. Hort Sci 41(3): 622-627.

10. Rouphael Y, M Cardarelli, E Rea, G Colla (2012) Improving melon and cucumber photosynthetic activity, mineral composition, and growth performance under salinity stress by grafting onto Cucurbita hybrid rootstocks. Photosynthetica 50(2): 180-188.

11. Liu YF, HY Qi, CM Bai, MF Qi, CQ Xu, et al. (2011) Grafting helps improve photosynthesis and carbohydrate metabolism in leaves of muskmelon. Inter j Bio Sci 7(8): 1161.
12. Karaca F, H Yetișir, I Solmaz, E Candir, S Kurt, et al. (2012) Rootstock potential of Turkish Lagenaria siceraria germplasm for watermelon: plant growth, yield and quality. Turkish J Agric and Forestry 36(2): 167177.

13. Mohamed FH, KA El Hamed, MWM Elwan, MNE Hussien (2014) Evaluation of different grafting methods and rootstocks in watermelon grown in Egypt. Scientia Hort 168: 145-150.

14. Gomez KA, AA Gomez (1984) Statistical procedures for agriculture research. International Rice Research institute Textbook 84-297.

15. Park Dong Kum Kwon, Joon Kook Lee, Jae Han Choi, Young Ha Lee, Soon $\mathrm{Gu}(2003)$ Effects of soil salinities on growth and fruit quality in oriental melon (Cucumis melo). J. the Korean Society for Hort Sci 44(5): 616-619.

16. Badr MA, SDA Hussein (2008) Yield and fruit quality of drip-irrigated cantaloupe under salt stress conditions in an arid environment. Australian J Basic and Applied Sci 2(1): 141-148.

17. Botia P, JM Navarro, A Cerda, V Martinez (2005) Yield and fruit quality of two melon cultivars irrigated with saline water at different stages of development. European J Agron 23(3): 243-253.

18. El Shraiy AM, M Mostafa, SA Zaghlool, S Shehata (2011) Alleviation of salt injury of cucumber plant by grafting onto salt tolerance rootstock. Australian J Basic and Applied Sci 5(10): 1414-1423.

19. Wadid MM (2002) Studies on the productivity and fruit quality of melon (Cucumis melo var. cantaloupensis) irrigated with saline or fresh Water in North Sinai Egypt. J Appl Sci 17(7): 728-746.

20. Moursy FS, MM Wadid, MM Hashim, AF Abou Hadid (2003) Studies on plant growth, productivity and quality of cantaloupe (Cucumis melo var. cantaloupensis) as affected by irrigation with saline water. Egyptian J Hort 30(1/2): 135-150.

21. Gurgel MT, CA Uyeda, HR Gheyi, FHT de Oliveira, PD Fernandes, et al. (2010) Growth of melon cultivated under saline stress and potassium doses. Revista Brasileira de Engenharia Agricola Ambiental 14(1): 3-10.

22. Rouphael Y, M Cardarelli, G Colla, E Rea (2008) Yield, mineral composition, water relations, and water use efficiency of grafted miniwatermelon plants under deficit irrigation. Hort Sci 43: 730-736.

23. Proietti S, Y Rouphael, G Colla, M Cardarelli, M Agazio, et al. (2008) Fruit quality of mini-watermelon as affected by grafting and irrigation regimes. J the Sci Food and Agric 88: 1107-1114.

24. Özmen S, R Kanber, N Sar, M Ünlü (2015) The effects of deficit irrigation on nitrogen onsumption, yield, and quality in drip irrigated grafted and ungrafted watermelon. J Integrative Agric 14(5): 966-976.

25. Hussien MN (2016) Effect of some genotypes on the response of grafted watermelon plants to abiotic stress. PhD. Thesi Hort Dep Agric Fac Suez Canal Univ Ismailia Egypt, Egypt, pp. 365.

26. Lily M Vargas Rodríguez, Jesús E Morales Barrera, José G Herrera Haro, Juan Antonio Bautista, et al. (1990) Effect of Citric Acid, Phytase and Calcium Levels on the Calcium and Phosphorus Content in Egg: YolkAlbumen and Shell, Yolk Color and Egg Quality in Diets of Laying Hens. Official methods of analysis. Association of Official Analytical Chemists $\left(15^{\text {th }}\right.$ edn) Washington DC, USA. 


\section{(C) This work is licensed under Creative}

To Submit Your Article Click Here: Submit Article

DOI: 10.32474/CIACR.2018.02.000128

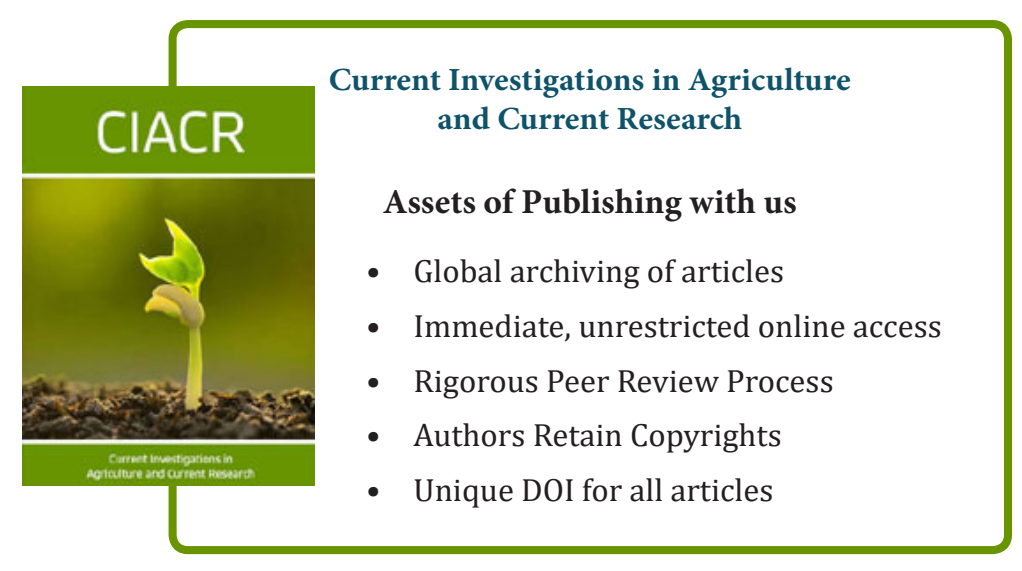

